\title{
Editorial
}

\section{Training programs and implementation process of positive youth development programs}

\section{Daniel T.L. Shek, Rachel C.F. Sun and Joav Merrick}

In the areas of adolescent development and adolescent psychopathology, there are many researchers arguing that developmental assets or psychosocial competencies are very important to adolescent development (1). Although positive youth development qualities have been conceived differently in the literature, it is commonly referred to the competencies of (a) self-understanding and cultivating discipline; (b) working with others, communicating, cooperating, negotiation, and building relationships; (c) coping, adapting, and being responsible; and (d) making good judgments, evaluating, making decisions, and problem-solving. According to the Collaborative for Academic, Social and Emotional Learning (CASEL: http:// www.casel.org), social and emotional learning covers emotional recognition and management skills, cultivation of care and concern for others, responsible decision-making, positive relationship building, and effective handling of life stresses.

With reference to the Chinese culture, because there is obsessive emphasis on academic excellence in adolescents, holistic development is normally not the focus of parents as well as young people (2). Furthermore, there are research findings showing that adolescents in Hong Kong faced high levels of stress in different psychosocial domains (3). Obviously, how to promote holistic development in Chinese adolescents and help them cope with life stresses are important issues to be considered. Unfortunately, there are few programs focusing on the promotion of psychosocial competencies in adolescents in Hong Kong.

To promote holistic adolescent development, The Hong Kong Jockey Club Charities Trust earmarked HK $\$ 400$ million for a positive youth development program entitled "P.A.T.H.S. to Adulthood: A Jockey Club Youth Enhancement Scheme". In the Tier 1 Program of the project, a curricular-based program for junior secondary school students (Secondary 1 to Secondary 3 levels in Hong Kong which is equivalent to Grade 7 to Grade 9 in the North American system) was designed based on the 15 positive youth development constructs identified from the existing successful positive youth development programs. The original phase of the program implementation was carried out in $2005 / 2007$ to $2008 / 2009$ school years. Because of the overall success of the original phase, the project was extended from 2009/2010 to 2011/2012 school years, with an additional $\mathrm{HK} \$ 350$ million earmarked for the project (4).

For each of the Secondary 1 to Secondary 3 programs, potential program implementers (mainly teachers and social workers) receive 20 hours of training before implementing the program in their schools. Generally speaking, there are 3 days of training at each grade. In addition to learning the conceptual foundation, program philosophy, curriculum issues and evaluation methods intrinsic to the project, teaching units in the curriculum, as well as the ways by which the program can be successfully implemented are covered in the training programs. One particular feature of the training program in the Project P.A.T.H.S. is that the potential program implementers are encouraged to reflect on their motivation to teach the program and appreciate the vision of the program. The training programs are designed according to the principles proposed in Shek and Wai (5).

In a series of papers documenting the evaluation of the training programs of the Project P.A.T.H.S., quantitative and qualitative research findings based on subjective outcome evaluation showed that the program participants generally had positive perception about the training program and the instructors (6-10). They also felt that the training promoted their understanding of the project and encouraged them for self-reflection. The findings echoed other qualitative evaluation findings suggesting that the training program was effective in promoting the participants' knowledge and attitudes about the program and their reflections about their teaching efficacy and skills. Additional qualitative data collected via reflective techniques similarly showed that most of the respondents had positive perception of the training program and instructors. Most of the participants felt that the training program enhanced their understanding of positive youth development and the project, and offered them opportunities for self-reflection. Participants showed more confidence in program implementation but they also pointed out difficulties in program implementation. Although the papers edited by Shek and Merrick (11) constituted pioneer work on the training programs of a positive youth development program in a Chinese context, there is a need to replicate the findings. As such, several papers documenting the effectiveness of the training programs for the Project P.A.T.H.S. are included in this special issue.

In addition to having quality training programs, it is also important to have a quality program implementation process. Unfortunately, in the context of adolescent prevention and positive youth development programs, most of the existing evaluation effort is placed on objective outcome evaluation and subjective outcome evaluation, and findings on program implementation quality are rarely reported. Scheirer (12) argued that there were several reasons for understanding the program implementation process. First, understanding the process can 
guard against type III error (i.e., existence or non-existence of program effect because of occurrence of activities different from those intended by the program developers). Second, feedback collected in the implementation process (such as appreciation and observation of positive changes in the program participants) can promote fidelity in the implementation process. Third, process evaluation can help program developers understand whether the intended targets receive the program. This point is important because program adherence and fidelity in program implementation are issues that should be seriously considered in the process of program implementation. Fourth, process evaluation can help to identify factors that contribute to program success. Finally, program developers can use process evaluation findings to understand how the developed program can be successfully implemented in human organizations and communities which are always complex in nature. Because there are few systematic studies investigating program implementation in the Chinese culture, papers on program implementation are included in this special issue. Previous studies on the implementation process of the Project P.A.T.H.S. have identified factors facilitating the implementation process which can lead to program effectiveness $(13,14)$.

Concerning the above background, this special issue contains several papers documenting the evaluation of training workshops and program implementation for the Project P.A.T.H.S. in Hong Kong. There are several unique features of this special issue. First, evaluation findings of the training programs are generally consistent with those reported in the previous studies. From a scientific point of view, replication of research findings is an important benchmark to be desired. In addition, the related papers are important because evaluation findings in positive youth development programs based on large samples are seldom reported in the literature. Second, findings on program implementation generally replicated those reported in the previous studies. Third, as there is a paucity of studies on adolescent prevention and positive youth development programs in Asia (see Shek and Yu's paper in this special issue), the papers in this special issue have academic and practical importance. Finally, this special issue is the second known scientific publication documenting training programs and program implementation related to positive youth development programs in different Chinese communities. It is our humble wish that this special issue can motivate colleagues to conduct more studies on training programs and program implementation of positive youth development programs in both Chinese and international contexts.

\section{References}

1. Lerner RM, Benson PL, editors. Developmental assets and assetbuilding communities: implications for research, policy, and practice. New York: Kluwer Academic/Plenum, 2003.
2. Shek DTL, Chan LK. Hong Kong Chinese parents' perceptions of the ideal child. J Psychol 1999;133:291-302.

3. Shek DTL. Social stress in Hong Kong. In: Estes J, editor. Social development index. Hong Kong: Oxford University Press, 2005:213-22.

4. Shek DTL, Sun RCF. Effectiveness of the Tier 1 Program of Project P.A.T.H.S.: findings based on three years of program implementation. Scientific World Journal 2010;10:1509-19.

5. Shek DTL, Wai CLY. Training workers implementing adolescent prevention and positive youth development programs: what have we learned from the literature? Adolescence 2008;43:823-45.

6. Shek DTL, Chak YLY. Design of training programs for a positive youth development program: Project P.A.T.H.S. in Hong Kong. Int J Adolesc Med Health 2010;22:345-67.

7. Shek DTL, Chak YLY. Training potential program implementers of the Project P.A.T.H.S. (Secondary 1 Program) in Hong Kong. Int J Adolesc Med Health 2010;22:369-84.

8. Shek DTL. Quantitative evaluation of the training program of the Project P.A.T.H.S. in Hong Kong. Int J Adolesc Med Health 2010;22:425-35.

9. Shek DTL, Wong KKL. Subjective outcome evaluation of the training program of the project P.A.T.H.S. based on qualitative findings. Int J Adolesc Med Health 2010;22:437-47.

10. Shek DTL, Wong KKL. Qualitative evaluation of the training program of the Project P.A.T.H.S. in Hong Kong. Int J Adolesc Med Health 2010;22:413-23.

11. ShekDTL, Merrick J, Eds. Special issue on positive youth development and training. Int J Adolesc Med Health 2010;22:341-447.

12. Scheirer MA. Designing and using process evaluation. In: Wholey JS, Hatry HP, Newcomer KE, editors. Handbook of practical program evaluation. San Francisco, CA: Jossey-Bass, 1994:40-68.

13. Sun RCF, Shek DTL, Siu AMH. Positive school and classroom environment: precursors of successful implementation of positive youth development programs. ScientificWorldJournal 2008;8:1063-74.

14. Shek DTL, Ma HK. Evaluation of the Project P.A.T.H.S. in Hong Kong: are the findings replicable across different populations? ScientificWorldJournal 2010;10:178-81.

Professor Daniel T.L. Shek, PhD, FHKPS, BBS, JP

Department of Applied Social Sciences, The Hong Kong

Polytechnic University, Hunghom, Hong Kong, P.R. China

E-mail: daniel.shek@polyu.edu.hk

Dr. Rachel C.F. Sun, PhD

Faculty of Education, The University of Hong Kong,

Pokfulam Road, Hong Kong, P.R. China

E-mail: rachels@hku.hk

Professor Joav Merrick, MD, MMedSci, DMSc

National Institute of Child Health and Human Development, Division for Mental Retardation, Ministry of Social Affairs, Jerusalem, Israel

E-mail: jmerrick@zahav.net.il 